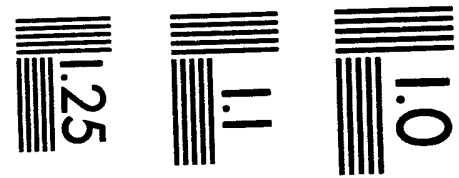

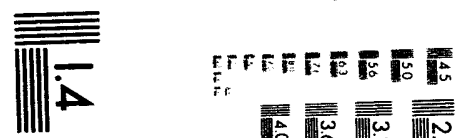

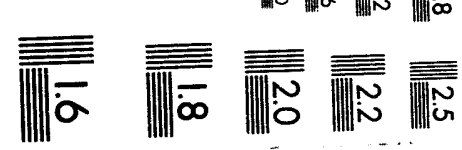



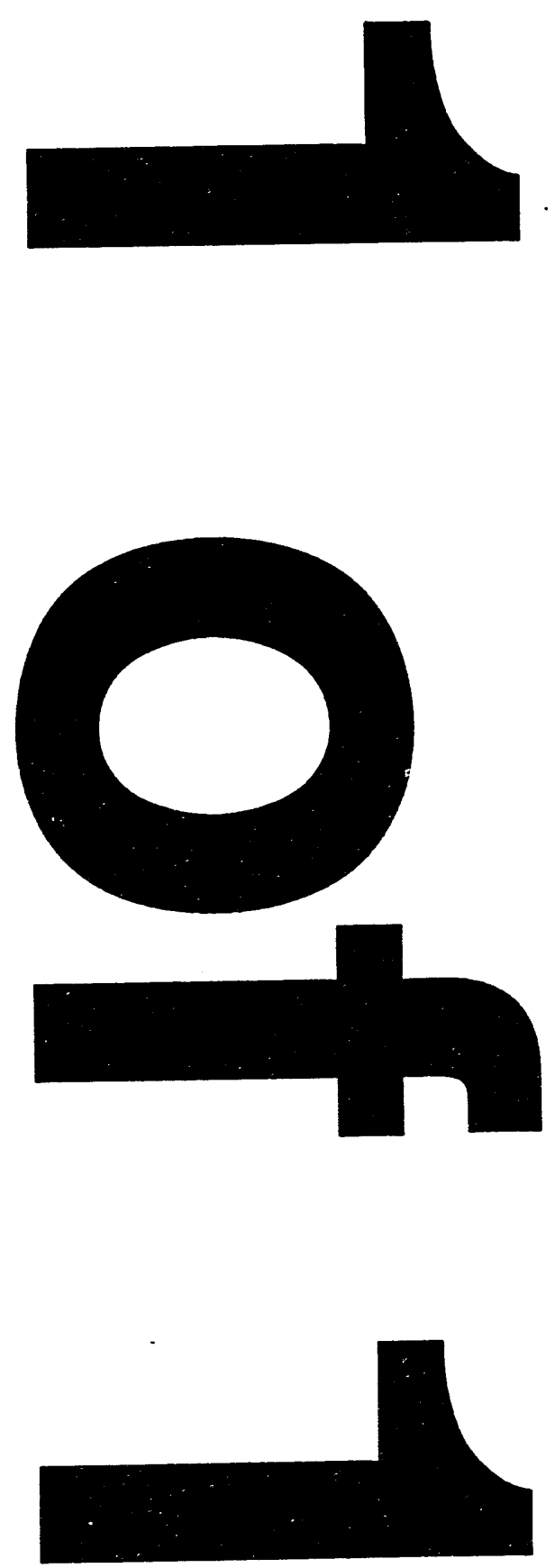
LA-UR- $94-526$

$$
\operatorname{con} 8-940226--4
$$
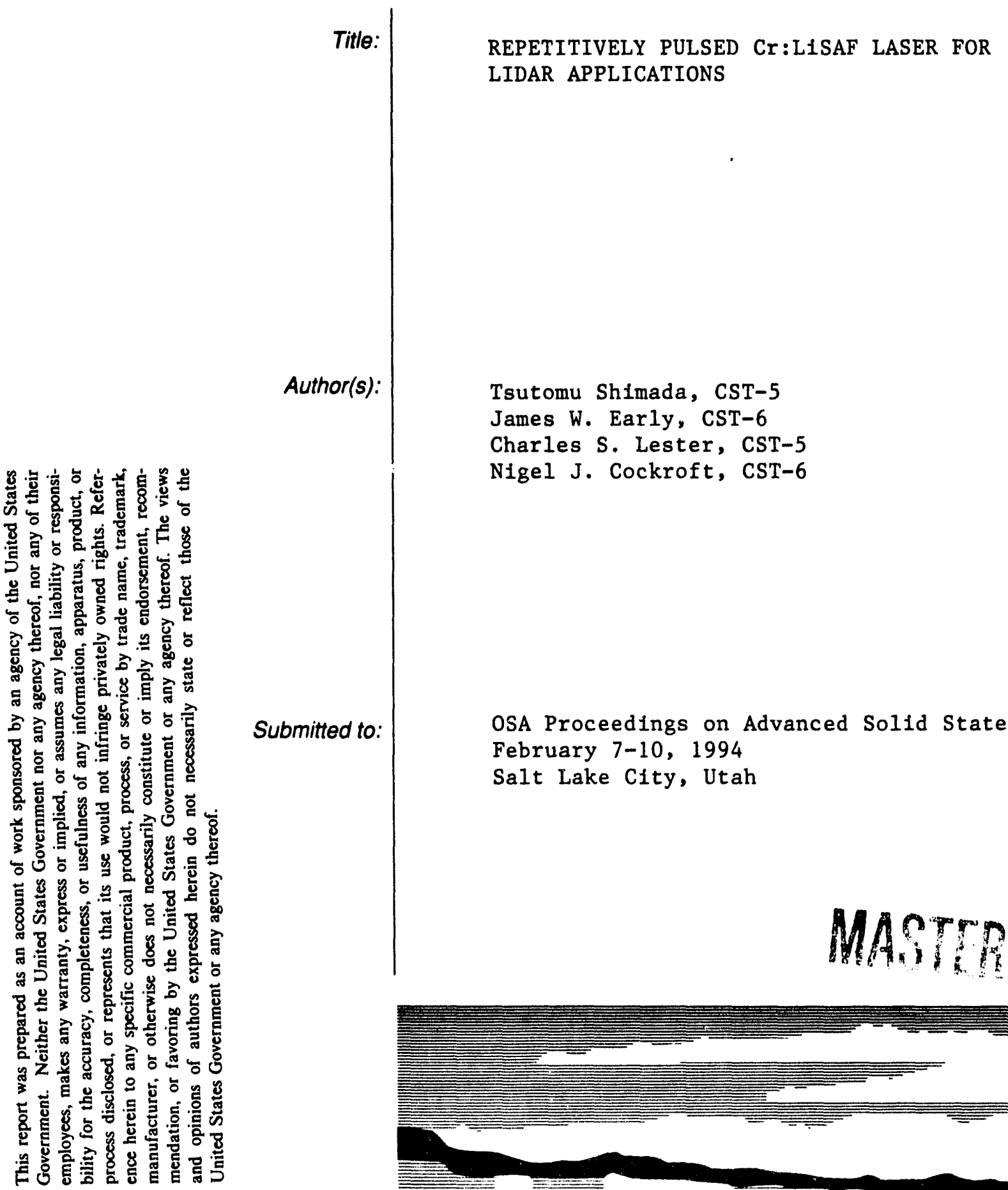

Submitted to:

OSA Proceedings on Advanced Solid State Lasers ('94) February 7-10, 1994

Salt Lake City, Utah

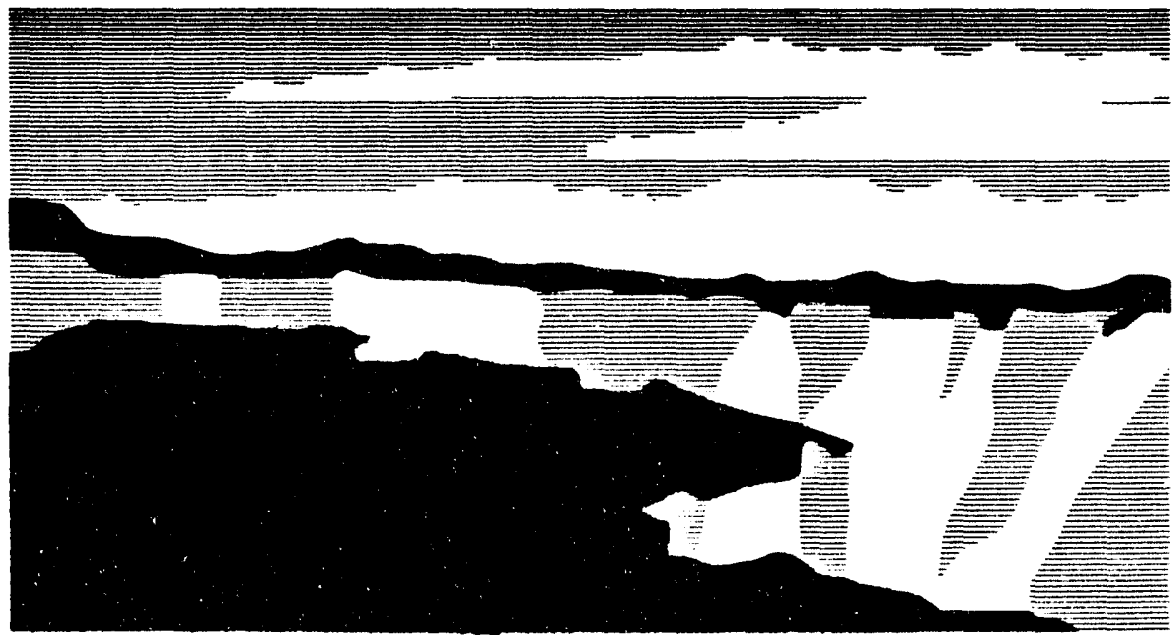

Los Alamos National Labsratory, an aftirmative action/equal opportunity employer, is operated by the University of California for the U.S. Department of Energy under contract W-74n5-ENG-36. By accoptance of this article, the publisher recognizes that the U.S. Government retains a nonexclusive, royalty-free license to publish or reproauce the published form of this contribution, or to allow others to do so, for U.S. Govemment puposes. The Los Namos National Laboratory requests that the publisher identify this anticle as work performed under the auspices of the U.S. Department of Energy.

$$
\text { db Form No. } 236 \text { R5 }
$$




\section{Repetitively Pulsed Cr:LiSAF Laser for Lidar Applications}

Tsutomu Shimada, James W. Early, Charles S. Lester and Nigel J. Cockroft

Chemical Science and Technology Division

Los Alamos National Laboratory

MS-E535, P.O.Box 1663

Los Alamos, NM 87545

Phone: (505)-667-8391

Fax: (505)-665-4637

This paper will be appearing in OSA Proceedings on Advanced Solid State Lasers ('94), Feb.7-10,1994, Salt Lake City,Utah. 


\title{
Repetitively Pulsed Cr:LiSAF Laser for Lidar Applications
}

\author{
Tsutomu Shimada, James W. Early, Charles S. Lester and Nigel J. Cockroft \\ Chemical Science and Technology Division \\ Los Alamos National Laboratory \\ MS-E535, P. O. Box 1663 \\ Los Alamos, NM 87545 \\ Phone: (505)-667-8391 \\ Fax: (505)-665-4637
}

\begin{abstract}
:
A Cr:LiSAF laser has been successfully operated at time averaged powers up to $11 \mathrm{~W}$ and at pulse repetition rates to $12 \mathrm{~Hz}$. During Q-switch operation, output energy as high as $450 \mathrm{~mJ}$ (32 ns FWHM) was obtained. Finally, line narrowed Qswitched pulses $(<0.1 \mathrm{~nm})$ from the Cr:LiSAF laser were successfully used as a tunable light source for lidar to measure atmospheric water content.
\end{abstract}

\section{Introduction:}

Cr:LiSAF as a flashlamp pumped laser material ${ }^{1}{ }^{-3}$ ) has received considerable attention recently due mainly to 1) its broad gain bandwidth which provides wide lasing wavelength tunability and ultrashort pulse amplification and 2) a reasonably long lifetime $(67 \mu \mathrm{s})$ which supports Q-switched operation.

In a particular application such as lidar for remote sensing, the laser is required to operate with a reasonable repetition rate to provide reliable information by averaging over many pulses. We have been developing a repetitively pulsed tunable Qswitched Cr:LiSAF laser system especially for lidar applications.

\section{Experiments:}

A Cr:LiSAF rod with a length of $10 \mathrm{~cm}$ and a diameter of $0.635 \mathrm{~cm}$ was used for these experiments. The rod was doped to a $\mathrm{Cr}$ concentration of $1.5 \%$. A dual xenon flashlamp pump cavity, containing a Pyrex insert surrounded by $\mathrm{BaSO}_{4}$ to provide a broadband, diffusively reflecting surface to the flashlamp light, was used to pump the rod.
The flashlamp power supply was designed to produce a $60 \mu \mathrm{s}$ (FWHM) wide current pulse into the two flashlamps connected in series. Pump length was limited to $8.9 \mathrm{~cm}(3.5 \mathrm{in})$ due to the dimensions of the pump cavity and the discharge gap of the flashlamps. The laser resonator consists of a $2-\mathrm{m}$ radius of curvature, high reflectivity, dielectric mirror and a flat, dielectric output coupler. A cavity length of $61 \mathrm{~cm}$ was used. We used 1 to 3 plate birefringent tuner for the wavelength tuning, Pockells'cell for Q-switching and 2 etalons for narrow linewidth operation. Block diagram of the laser setup with all the intracavity optics are shown in Fig.1.

It should be noted that we used a pH-7 buffer solution as a cooling water to minimize the weight loss of the Cr:LiSAF crystal due to the dissolution to the cooling water, and we have not seen any measurable weight loss of the Cr:LiSAF crystal over six month period.

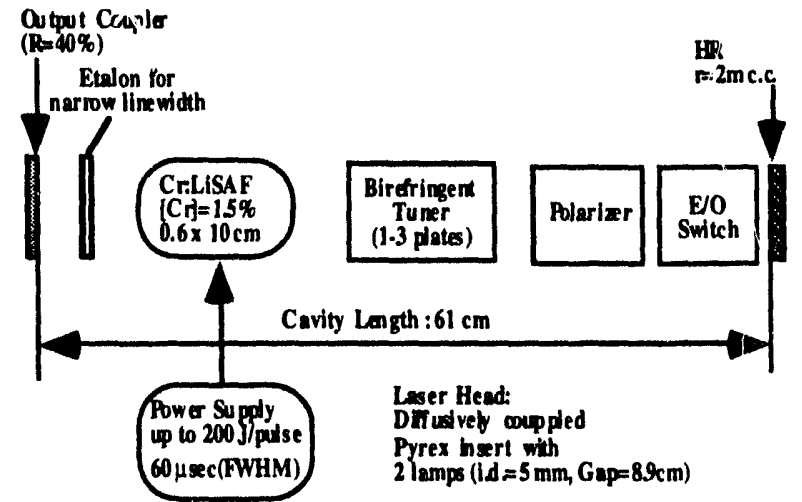

Figure 1. Block diagram of the typical laser setup when all the intracavity optics are placed. 


\section{Results and Discussions:}

\section{i) Free Running Mode}

Figure 2 shows the dependence of laser output pulse energy on the stored electrical energy at the capacitor for several different refrectivities of output coupler. The laser was operated at a $1 \mathrm{~Hz}$ repetition rate in the free lasing mode at a wavelength of $853 \mathrm{~nm}$ without any intracvity dispersive elements. As shown in the Fig.2, increasing the reflectivity of the optical cavity, decreases oscillation threshold input energy, whereas, slope efficiency becomes maximum at a output coupler refrectivty about $60 \%$. A maximum output energy of $3.1 \mathrm{~J}$ was obtained with an electrical energy input to the flashlamp of $80 \mathrm{~J}$. A maximum slope efficiency of $4.9 \%$ and an over all efficiency of $3.8 \%$ was obtained using the $60 \%$ reflective output coupler. Maximum laser output energy obtained shown in Fig. 2 is not limited by the optical damage of the crystal nor the optical elements of the cavity used, so that one will be able to obtain much higher output energy and better overall efficiency with a higher input energy. Typical waveforms observed for free running mode had a gain Q-switched pulse followed by a 30-50 $\mu$ s long relaxation rscillation depending on the pump energy.

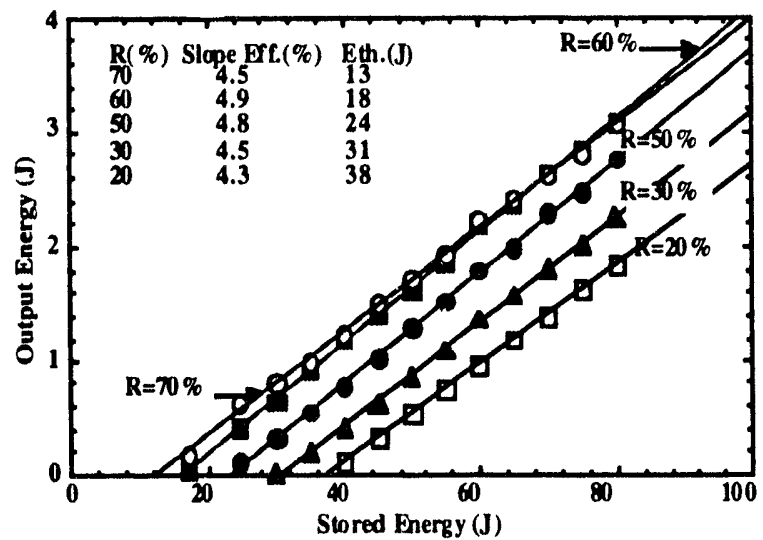

Figure 2. Output energy dependence on stored energy for various refrectivities of output coupler. (free running mode, @ 853 nm)

We have also measured the dependence of average laser output power on pulse repetition rate for electrical energy input to the flashlamps of $40 \mathrm{~J} / \mathrm{pulse}$ and 75 J/pulse (see Fig.3). When compared to the output pulse energy at a $1 \mathrm{~Hz}$ rate, we typically observe a reduction in pulse energy at higher pulse rates for both input energy case as shown in Fig.3. However, compared to the output energy at a $1 \mathrm{~Hz}$ pulse rate, the pulse energy at $5 \mathrm{~Hz}$ dropped to $80 \%(2.05 \mathrm{~J}$ compared to $1.64 \mathrm{~J})$. At a higher pump energy of $100 \mathrm{~J}$, an average output power of $11 \mathrm{~W}(2.2 \mathrm{~J} /$ pulse $)$ was obtained during $5 \mathrm{~Hz}$ operation (a reduction in pulse energy of $24 \%$ from that measured at $1 \mathrm{~Hz}$ ). We also operated the laser at repetition rates up to $12 \mathrm{~Hz}$ with a lower pump energy of $40 \mathrm{~J}$. With the same cavity optics, a maximum average output power of $4.3 \mathrm{~W}$ was obtained (a $37 \%$ reduction in pulse energy compared with the $1 \mathrm{~Hz}$ operation). We attribute the decrease in pulse energy at higher repetition rates to increased diffraction losses within the cavity due to thermal lensing in the $\mathrm{Cr}: \mathrm{LiSAF}$ rod, which can be compensated by the proper cavity design. Although energy lifetime shortening and subsequent gain reduction in the Cr:LiSAF rod can occur at high thermal loading, measurements of the small signal gain as well as the fluorescence waveform show no dependence on repetition rate up to our maximum time averaged pump power of $480 \mathrm{~W}$.

After the repetitive high average power experiments, we observed a couple of micro-crack fomations in the Cr:LiSAF crystal due to the high thermal loading. This thermal fracture considered to be occured during average flashlamp input power of $480-500 \mathrm{~W}$. In spite of the micro-crack formation, we could not see any changes in the laser performances during experiments.

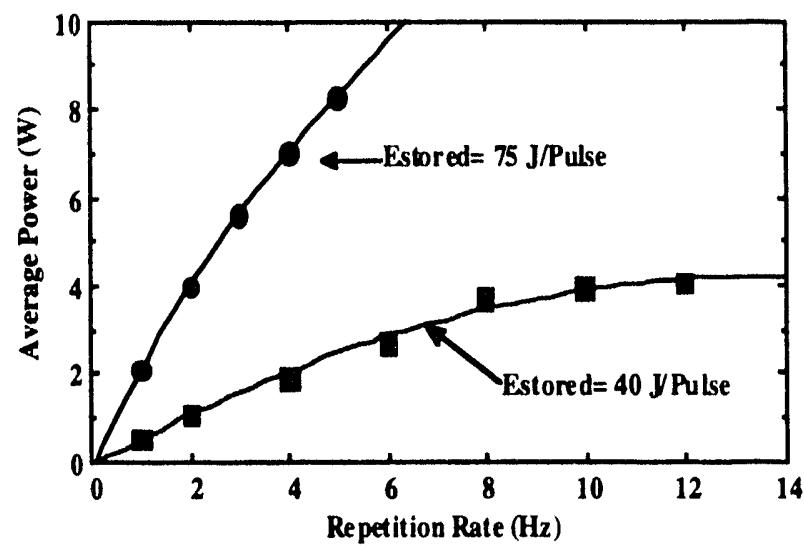

Figure 3. Average power dependence on repetition rate for diffrent stored energy(free running mode @853 nm). Maximum average power obtained was $11.3 \mathrm{~W}$, (Estored $=100 \mathrm{~J}, 5 \mathrm{~Hz}, \mathrm{R}=40 \%$ ).

Spectral measurements of the flashlamp's output transmitted through the Pyrex insert of the pump chamber showed a peak emission at the wavelength of $450 \mathrm{~nm}$. The peak wavelength was found to be independent of lamp energy over the $35-100 \mathrm{~J}$ range of lamp energy investigated. This is due mainly to the $350 \mathrm{~nm}$ uv transmission cut-off which is exhibited by Pyrex insert. We did observe, however, a rapid increase in the emission at $450 \mathrm{~nm}$ relative to that at $650 \mathrm{~nm}$ as 
lamp energy increased. This may result adversely in increased thermal loading to the Cr:LiSAF crystal due to multiphonon relaxation between the ${ }^{4} \mathrm{~T}_{1} \mathrm{a}$ and ${ }^{4} \mathrm{~T}_{2}$ levels. Considering the lower thermal fracture limit of Cr:LiSAF crystal $(4.5 \mathrm{~W} / \mathrm{cm}) 4$ ), improvements of the flashlamp color temperature match with the $\mathrm{Cr}$ absorption bands, especially to the $650 \mathrm{~nm}$ band, is needed to reduce a thermal loading for the higher average power operation. This may also be accomplished without significant degradation to the pumping efficiency by utilizing a large bore flashlamp and/or fluorescence converter.

\section{ii) Q-Switched Mode}

In addition to the repetitive, free-lasing operation of the Cr:LiSAF laser, we have successfully demonstrated Q-switched lasing. Q-switched pulses with an energy of $450 \mathrm{~mJ}$ and a pulsewidth of $32 \mathrm{~ns}$ (FWHM) were obtained during $1 \mathrm{~Hz}$ operation for an electrical input of $45 \mathrm{~J} /$ pulse to the flashlamps. Output energy stability was better than $5.0 \%$. The Q-switched laser was also operated at a repetition rate of $5 \mathrm{~Hz}$ to provide an output energy of $90 \mathrm{~mJ} /$ pulse at a lower pump energy. However, we observed optical damage in the form of seriesed void formation in the crystal as Richardscn et.al. reported ${ }^{5)}$ when the electrical input energy to the flashlamp was increased to $50 \mathrm{~J}$.

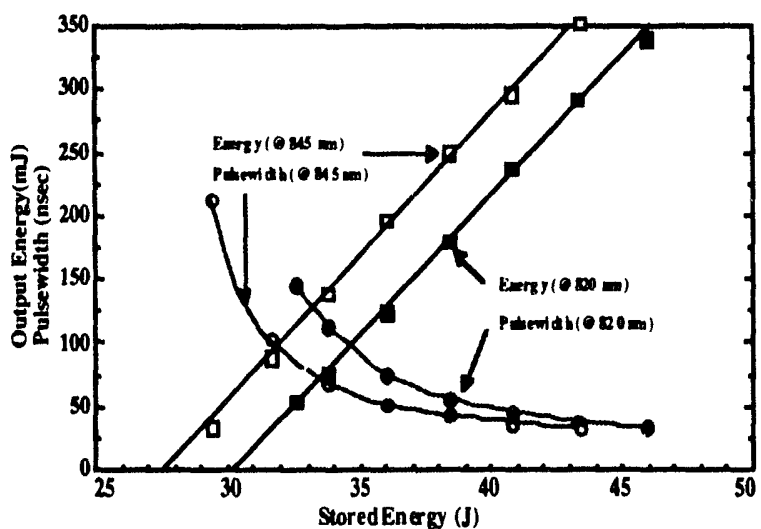

Figure 4. Q-switched output energy and pulsewidth dependence on stored energy for diffrent wavelwngth. (with a birefringent tuner without etalon).

Tuning of the laser's output was also demonstrated over the wavelength range of 820 to $890 \mathrm{~nm}$ using a single and triple plate birefringent tuner, and this was limited by the coating of the optics used for the laser cavity centered at $850 \mathrm{~nm}$. Figure 4 indicates Q-switched output energy and pulsewidth dependence on the stored energy at two different wavelengths. Since small signal gain peak locates in the vicinity of $850 \mathrm{~nm}$, higher output energy and shorter pulse width were obtained at $845 \mathrm{~nm}$ than at $820 \mathrm{~nm}$ with a same stored energy. However, pulsewidth at both wavelengths were to be the close value of $30 \mathrm{~ns}$ when the laser was operated at stored energy of more than $40 \mathrm{~J}$. Typical linewidth with a birefringent tuner was $3 \mathrm{~nm}$ for single plate tuner and $0.7 \mathrm{~nm}$ for 3 plate tuner, respectively. Linewidth as narrow as less than $0.1 \mathrm{~nm}$ was measured with an additional set of intracavity etalons.

\section{iii) Diode Injection Seeded Q-Switched Mode}

For further narrower linewidth operation, we have worked on single mode diode injection seeding experiment. A single mode CW output (@890 nm, 8 $\mathrm{mW}$ ) from the diode laser was introduced into a Cr:LiSAF laser cavity through the output coupler. Cr:LiSAF laser for this experiment consists of an output coupler( $\mathrm{R}=40 \%), 1 \mathrm{~mm} \phi$ aperture for a single transverse mode, Cr:LiSAF rod, E/O switch, polarizer, birefringent tuner and $\mathrm{HR}(2 \mathrm{~m}$ C.C $)$.

To avoid a optical damage and wavelength instability of the diode laser due to the feed back light from the Cr:LiSAF laser, we used a pin hole spatial filter and 2 Farady isolators in series $(2 \times 30 \mathrm{db})$. Spatial filter also vias used to match the mode sizes of the two lasers. By carefully observing the Q-switched laser waveforms with injection, we could see the two mode beating with a frequency of longitudinal mode spacing (modulation depth of $30-40 \%$ ) at the intracavity injection power range from $40 \mu \mathrm{W}$ to $330 \mu \mathrm{w}$. Although we did not measure the linewidth precisely, an eatalon with 30 $\mathrm{GHz}$ free spectral rage showed $\mathrm{Cr}$ :LiSAF laser linewidth was comparable to that of single mode diode laser we used. Typical laser output energy during this experiment was $10 \mathrm{~mJ} /$ pulse with a pulsewidth of $50 \mathrm{~ns}$ (FWHM).

It may be well worth to mention that we obserbed optical damges during Q-switch operation; one case with a multiple transverse mode operation without intracvity apperture at an output energy of more than $450 \mathrm{~mJ}$ (beam diameter : $6.35 \mathrm{~mm} \phi$ ) as mentioned before, the another case with a single transverse mode operation at an output energy of $12 \mathrm{~mJ}$ (beam diameter : $1.0 \mathrm{~mm} \phi)$. For both cases, a damage threshold fluence was about $1.5 \mathrm{~J} / \mathrm{cm}^{2}$ for the laser pulses with a pulsewidth of 30-50 ns(FWHM).

\section{iv) Cr:LiSAF Lidar Demonstration}

A LANL miniature lidar system which normally operates at $1.06 \mu \mathrm{m}$ was used as the Cr:LiSAF lidar receiver. Laser photons scattered backwards towards the lidar by the atmospheric aerosol particles are collected by an 8 " diameter, f/10 telescope and focused onto a silicon avalanche photodiode(APD) mounted on the back of the telescope. A 10-nm bandpass filter(at 820 - or $830-\mathrm{nm}$ ) was used to block out solar background, and this allowed night and day time operation. In this configuration, the miniature 
lidar telescope was aligned with the optical axis of the Cr:LiSAF transmitter giving a single line-of-sight with which to make measurements. By recording single lineof-sight duta as a function of time(referred as "timedomain" scans) one can monitor the temporal changes in atmospheric aerosol load over the spatial range of interest. This lidar system was operated to evaluate its performance characteristics such as (1) determining the lidar detection range and sensitivity by monitoring atmospheric changes at a fixed wavelength, and (2) measuring the atmospheric water content by tuning the laser wavelength in 818 to $870 \mathrm{~nm}$ region.

With transmitter output pulses of $100 \mathrm{~mJ}, 50$ ns (FWHM), we could detect the back scattered signal as far as more than $100 \mathrm{~km}$ during the daytime with a relatively high elevation angles. For the narrow linewidth transmitter configuration $(35 \mathrm{~mJ}, 65 \mathrm{~ns}$, linewidth $<0.1 \mathrm{~nm}$ ), measured results indicate return signal at $820.1 \mathrm{~nm}$ (on resonant to the water absorption line) from the mountain at $12 \mathrm{~km}$ was about $1 / 16$ of the signal measured at $824.0 \mathrm{~nm}$ (off resonant to the water absoption line) whereas measurements with a line width of $0.7 \mathrm{~nm}$ gives an extinction of only $1 / 2$. Further lidar experiments are now under way.

Table 1. Summary of the demonstrated values of Cr:LiSAF laser system.

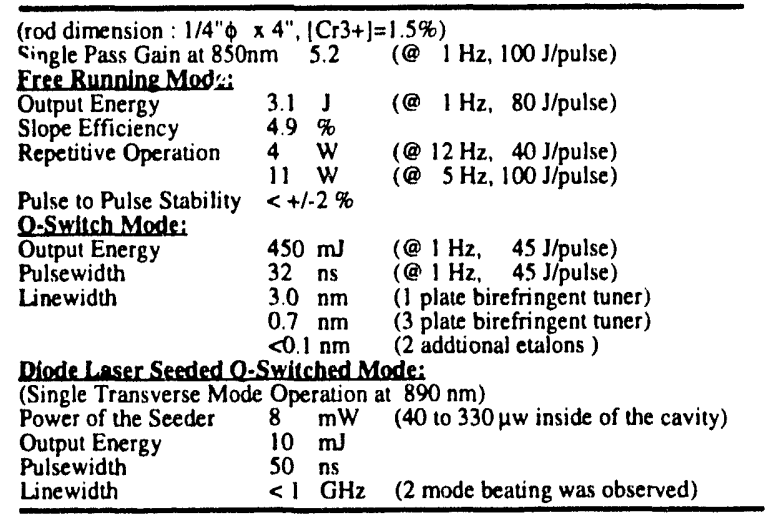

\section{Conclusions:}

We have successfully designed and developed a repetitively pulsed, tunable, Q-switched Cr:LiSAF laser system for lidar applications, and performances of the Cr:LiSAF laser system are summarized in table 1. To our knowledge, this is the first flashlamp pumped $\mathrm{Cr}:$ LiSAF laser system used as a tunable light source for lidar application. We observed a thermal fracture of the Cr:LiSAF rod in the form of micro-crack fomrations with a average flashlamp input power of $\sim 500 \mathrm{~W}$. Optical damages were obseved in the Cr:LiSAF crystal during a Q-switch operation(pulsewidth : 30 50 ns) and its threshold fluence was about $1.5 \mathrm{~J} / \mathrm{cm}^{2}$. For the higher average power and/or higher intensity $Q$ switched operation, design parameters such as thermal lensing effect, thermal fracture limit and optical damage threshold value are the ones of the most important issues for a $\mathrm{Cr}$ :LiSAF laser system design.

\section{Acknowledgments:}

The authors wish to thank to George Busch for his useful discussions on laser design. The authors also wish to thank to Joe Tiee and Robert Quick for the $\mathrm{Cr}$ :LiSAF laser demonstration for lidar measurements of atmospheric water contents and to David MacPherson for his collaboration on diode laser injection seeding experiment.

\section{References:}

1) M. Staldder, B. H. Chai, and M. Bass, Appl. Phys. Lett. 58, 216(1991).

2) T. Ditmire and M. D. Perry, Opt. Lett. 18, 426(1993).

3) P. Beaud, M. C. Richardson, E. Miesak, and B. H. Chai, Conference on lasers and Electoro-Optics 1993, CTuH3, Baltimore, Maryland, May 2-7(1993).

4) F. Hanson, C. Bendall, and P. Poirier, Opt. Lett.18, 1423(1993).

5) M. Richardson, M. J. Soileau, R. Beaud, R. De Salvo, S. Garnov, D. J. Hagan, S. Klimentov, K. Richardson, M. Sheik-Bahae, A. A. Said, E. Van Stryland and B. H. T. Chai, SPIE Proc., vol. 1848, 392(1992). 

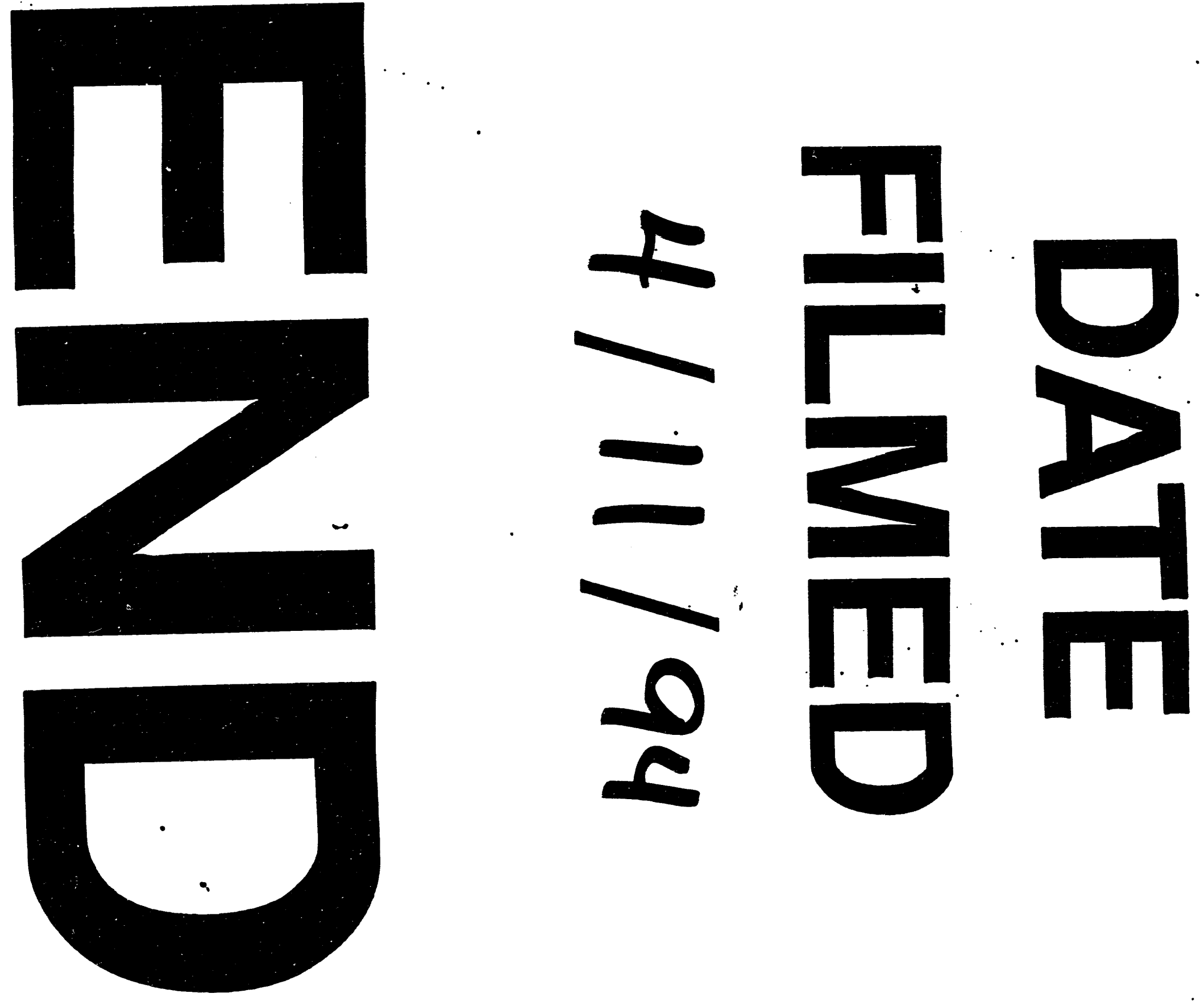
